\title{
Assessing Staff Alignment in Technical Services
}

\section{Jim LeBlanc and Martin Kurth}

Technical services units have routinely kept detailed monthly and annual statistics to assess productivity and efficiency, especially regarding functions that are easily measured. However, with the evolution of library user behavior, libraries' strategic directions, and technical services' practices, these numbers have become less and less useful in revealing the value of this work. In this paper, the authors introduce a methodology and draft model with which technical services managers can better assess not only their unit's productivity and efficiency, but the extent to which its activities align with a library's strategic values and the behavior of its users.

T $\mathrm{n}$ a summary of a discussion that took place at the 2013 American Library 1 Association Annual Conference, Winjum reported on concerns shared by many technical services managers regarding the challenge of demonstrating the impact of technical services' work on library operations in general. ${ }^{1}$ Indeed, the common decades-old practice of collecting detailed monthly and annual production statistics for those technical services tasks that are easily measurable has not changed much with time, even as user needs and interests and libraries's strategic goals have evolved. While the occasional practice of incorporating staff cost data into the equation certainly enhances the analytic value of raw production numbers, these more elaborate time- and activity-based cost studies aim almost exclusively at measuring productivity and efficiency. ${ }^{2}$ They do not address more user-oriented aspects of overall technical services' output, such as effectiveness, or the "quality processing completed in a timely manner," for which some libraries have undertaken separately focused analytical methods to account for such factors as the presence or absence of backlogs, or high or low throughput time. ${ }^{3}$ These figures do not reveal much about the relationship between the activities measured and user behavior, or the extent to which technical services' effort and priorities are aligned with a library's strategic vision (an increasing emphasis on the acquisition and licensing of electronic resources, for example). ${ }^{4}$ To remain relevant, these studies must be repeated as the circumstances of production change or evolve, such as the migration to a new integrated library system or increased automation and outsourcing. ${ }^{5}$ Additionally, while the data derived from existing assessment practices and carefully constructed cost studies is often reliably useful in reflecting a unit's productivity and efficiency, conducting reiterative cost studies can be costly — not to mention the intangible tax they impose on staff goodwill from having to repeatedly perform the task of recording and reporting their worktime based on function. Kaplan and Anderson acknowledged the role of these factors in their revised approach to activity-based costing in industry. ${ }^{6}$ While the elements of their costing formula do not vary 
significantly from those used in time-driven, activity-based technical services cost studies, they recommend abandoning the employee-survey model for "informed managerial estimates" based on unit-level figures. ${ }^{7}$ They also caution against the tendency to be "overly sensitive to small errors" in these estimates: "precision is not critical; rough accuracy is sufficient." Although these caveats may be construed as integral to a streamlining of the cost-study apparatus (i.e., a cost-cutting measure applied to the analytic process of assessing costs), they reveal a simple, practical path around the common obstacles to conducting repeated, rigorous cost studies of technical services activities. They also suggest important considerations for the design and implementation of richer approaches to evaluating the impact of library technical services' work. ${ }^{9}$

The aim of the current study is to propose a new way of thinking about staff allocation in technical services, not only in terms of productivity and efficiency, but in relation to a library's stated goals and its users' demonstrated needs and interests. It presents a methodology and model for reviewing activity-based cost figures in a simple and significantly broader way than is provided by existing methods. It is important to note that this model does not seek to replace existing means of compiling statistics and analyzing production trends, but to expand the scope of this analytic framework into a context in which the value of technical services performance can be more explicitly linked to the nuances of a library's central mission, especially in regard to its collections and their use.

\section{Method}

Rather than addressing commonly targeted aspects of technical services performance, such as productivity, efficiency, and effectiveness, this study aims to introduce a means to empirically evaluate the relationship between the alignment of technical services staff resources with collection policies and use patterns. Further, it considers the importance of using "informed managerial estimates" based on reliably derived data plus a willingness to regard the impact of these estimates with "rough accuracy" to permit easy, periodic reapplication of the assessment instrument (annually, semi-annually, or even more frequently). It is less a formal method for reporting statistical data than a tool for disclosing change in the strategic implications of staff alignment over time and eliciting increased managerial interest in regular, more focused reflection on the factors contributing to this change. It is important to note that this instrument is not intended as a benchmarking tool for comparing alignment across institutions, but as a customizable way to track staff and priority alignment within individual institutions. This last caveat is based on the assumption that

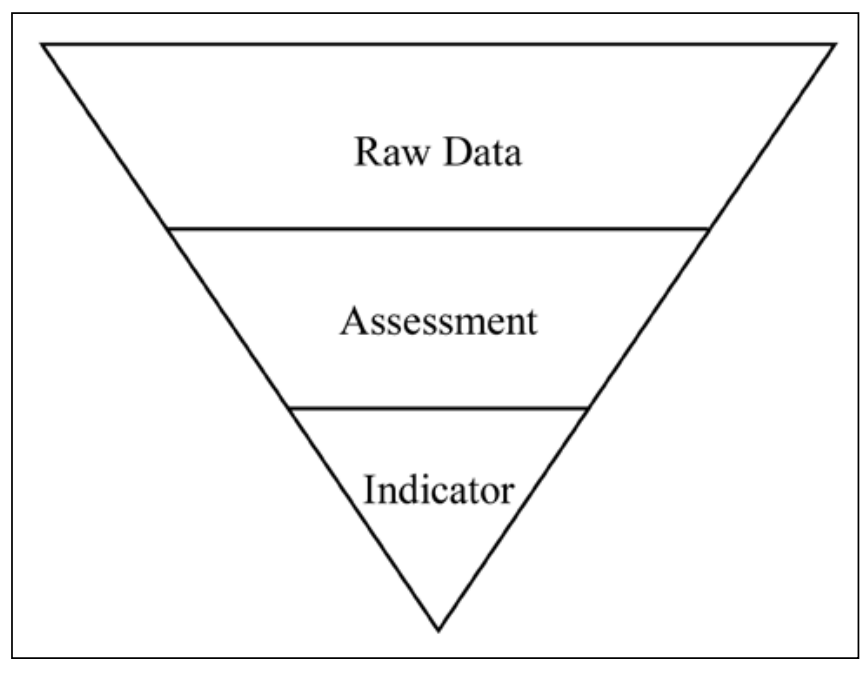

Figure 1. Inverted assessment pyramid.

harmonizing empirical inputs from multiple institutions in a comparative manner would undermine the ability to use the model frequently enough to reflect trends and longitudinal change.

Typically, the assessment of technical services functions begins with raw data such as annual production statistics or, more generally, opinion-based evaluative input from staff or library users. From this data, technical services managers draw conclusions and accordingly adjust practices and/or staff allocations. At a minimum, they will internalize these conclusions for planning purposes. Ideally, the collecting and analysis of this data allows technical services units to demonstrate their importance to library administrators. ${ }^{10}$ What is best for these purposes is evidence for and an understanding of these measurements over time, and this requires an instrument that supports an analytical perspective that is easy to reiterate. It is also important to include the relationship between technical services operations and collection policy and use.

To ensure a more holistic context for this kind of assessment, the current model challenges the traditional approach to interpreting technical services statistics. Rather than deriving a service-oriented conclusion from raw production numbers, the authors advocate for approaches that begin with an empirical, service-oriented conclusion, in the form of a single numerical indicator, to inform a broader understanding of the alignment of technical services staffing and performance with library-wide concerns. This approach is best illustrated by an inverted pyramid, with a top layer of raw data, which is distilled through model-driven mathematical factoring, into a single alignment indicator (see figure 1). ${ }^{11}$

The indicator that is generated by the tool becomes the initial focal point for the assessment, rather than the 
collection of raw data that traditionally serves as the ground for the evaluative activity.

\section{The Model (Structure)}

To demonstrate the applicability of the theoretical framework for evaluating the impact of technical services within a broader context, the authors present the following prototype for assessing staff alignment within technical services. Managing the scope of such a model to afford easy, reiterated application and to avoid levels of complexity is crucial to its utility. It is important to delimit the model's scope and to define its focus carefully. The benefits of this model derive less from the comprehensiveness of its individual elements than from the provocative power of its singular indicator, which is designed to steer the process of assessment in meaningful directions driven more by staff alignment than by more traditional measurements such as productivity, efficiency, and effectiveness, without marginalizing these factors.

For this study, the authors have chosen the acquisition and cataloging of non-serial titles in physical formats as the primary production focus to illustrate how to use the model. There are two reasons for this choice. First, the units of measurement related to these functions are longestablished and are regularly collected at most institutions. Second, the acquisition and cataloging of physical formats is an area of activity that many libraries have targeted for reduction as electronic formats usage continues to rise, both as actual downloads and as a percentage of total use of all library resources. ${ }^{12}$

The authors' tool is mathematical and uses an Excel spreadsheet to record and manipulate raw data on the acquisition and cataloging of titles in physical formats. Two detailed views of this model in spreadsheet form are provided in the supplementary materials to this paper. Table 1 contains sample data from a fictitious large university library for a two-year period. Table 2 shows that same data with the outputs displayed as the formulas used to generate these outputs. Read horizontally, these tables contain: (a) the categories measured, (b) the variable data for each category as input by the institution using the model, (c) the formulaic adjustment of that data to generate the alignment indicator that the model is designed to produce, (d) the variable data for a second year, (e) the adjusted values for this second-year data, and (f) a space for institution-specific notes. The spreadsheet framework enables the optional creation of additional columns to measure and compare data for subsequent years beyond the two years illustrated here. This structure also allows for customization.

The categories to be measured, as laid out vertically from top to bottom, support three key composite

$$
\underset{\text { transactions }}{\text { adjusted }}=\underset{\text { titles }}{\text { processed }} * \begin{gathered}
\text { contributed } \\
\text { value factor }
\end{gathered} * \begin{gathered}
\text { relative } \\
\text { cost factor }
\end{gathered}
$$

Figure 2. Adjusted transactions.

calculations. The first number that the model aims to generate is the efficiency quotient (row 65 in the model). This represents the total adjusted acquisitions and cataloging transactions per full-time equivalent (FTE) staff per 1,000 items. The adjusted acquisitions and cataloging transactions (cells C56 and E56) are the product of three variables: the actual number of titles processed in acquisitions and cataloging, the contributed value factor as applied to the category of material processed, and the relative cost factor based on the level of staff performing each transaction (see figure 2).

To perform the first step of this calculation, the actual number of titles processed (rows 4 and 5) is broken down by the type of material handled and the levels of staff who process it (rows 9-34). These last two factors are used to weigh the acquisitions and cataloging transactions according to values assigned by the individual institution using the tool.

The contributed value factor is based on a distinction between commonly held titles and those that are unique or rare. These types, "commodity" and "rare," are derived from terms applied by Dempsey, Malpas, and Lavoie to describe categories of library resources and their relative values within collections. They define commodity items as those non-unique materials that are "widely published or available through many channels"; rare items, conversely, are "unique" and "tend to be in one collection only."13 By adapting this nomenclature for technical services activities, the authors have divided the processing of commodity material into two types, "commodity (copy)" and "commodity (original)," to distinguish between those commonly held titles that are accessioned into local collections using existing catalog copy and those commodity items that are processed without pre-existing copy (i.e., through original cataloging). While some proportion of items in this latter subcategory may be unique, most of these titles are commodity material that has not yet been accessioned into other libraries' collections. Although these particular resources may have no greater value within an individual library's collection, the original cataloging provided by the first institution to handle them adds value from a processing perspective that lowers processing costs for other institutions. These contributed value factors appear in rows 38-40 of the table.

The relative cost factor further influences the weighted adjustment of the raw number of acquisitions and 


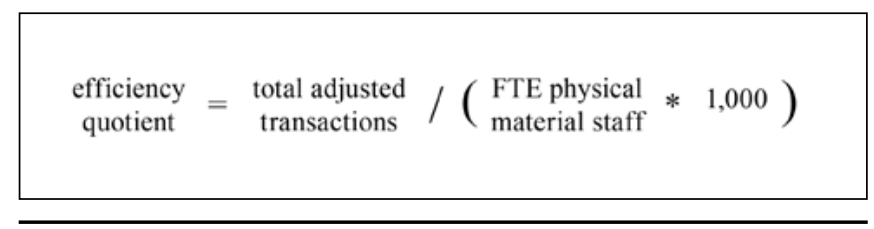

Figure 3. Efficiency quotient.

cataloging transactions by incorporating staffing levels, or more precisely, the typical costs (salary, plus benefits) related to the staff who perform the transaction, into the equation. The costs of four levels of staff (including one for students) appear in rows 44-47 of the table. The relative cost factors, comprising a comparison of the four cost levels expressed as ratios, appear in rows 51-54. The model assumes that the lower the staffing costs for each type of transaction counted, the higher the cost efficiency of the technical services unit as a whole.

This three-part weighted measurement of the technical services unit's productivity represented as total adjusted transactions is used as the dividend in the numerical relationship that produces the efficiency quotient (row 65). As explained above, the divisor in this equation is the total number of FTE staff allocated to these tasks per 1,000 items processed (see figure 3).

The second key composite calculation expressed in the model is the usage value multiplier, which appears in row 83 of the table. This number reflects the gap between the proportion of total non-managerial technical services staff devoted to the acquisition and cataloging of monographic material in physical formats (row 69) and the percentage of collection use generated by these resources (row 79). The difference between these two percentages reflects the extent to which the allocation of technical services staff effort to the processing of these items is aligned with the overall use of material in these formats (see figure 4).

This is an admittedly crude way to generate what purports to be a precise number. Assuming that: (1) the labor required to process material in physical formats is greater than that required to process titles in electronic formats, and (2) the use of the material in the latter format (represented by number of downloads) is indeed growing, then the smaller this gap, the greater the alignment of technical services staffing with user behavior. As in the formula for weighting productivity (i.e., in the total adjusted ACQ/CAT transactions), the contributed value factor for special/rare material (row 40) is applied here to reflect the added import of this rare and unique material to library users.

When combined with the efficiency quotient, the usage value multiplier contributes to the generation of a figure that integrates the measurement of productivity, efficiency, and the alignment of this work with a library's strategic collecting goals and the behavior of its users regarding the

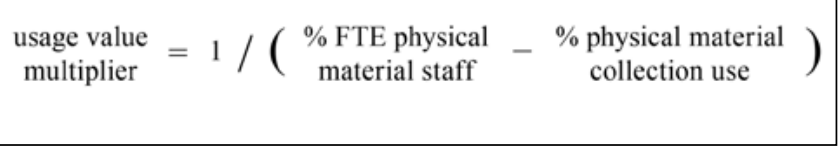

Figure 4. Usage value multiplier.

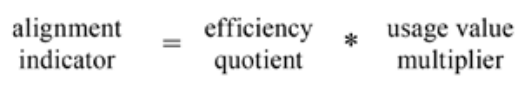

Figure 5. Alignment indicator.

collection as a whole. ${ }^{14}$ This figure, the alignment indicator (row 85), is the third important composite calculation performed in the model. As the single numeric product extracted from the table's inputs and equations, it represents the instrument's key output. The alignment indicator is the product of the efficiency quotient times the usage value multiplier, the latter of which is expressed as an inverse ratio (see figure 5). ${ }^{15}$

In this way, the alignment indicator integrates a technical services operation's productivity and efficiency with the strategic importance of the material processed by that unit and the anticipated value of this work to the library's user community.

\section{The Model (Application)}

To demonstrate how this assessment method works, the authors have populated tables 1 and 2 with sample data from a fictitious large research library. Column B contains the institution-specific variables for the first-year of the model's use. Column $\mathrm{C}$ shows the weighted recalibration and recombination of these variables for this same year, based on the principles elaborated in the previous section.

During the first sample year, the technical services unit acquired 40,000 new titles; it also cataloged 45,000 new titles (rows 4-5, column B) ${ }^{16}$ The detailed breakdown of this processing (rows 9-34) shows that 35,000 of the new titles acquired fall into the category of commodity (copy), 3,000 into commodity (original), and 2,000 into special/ rare. Forty thousand of the new titles cataloged fall into the category commodity (copy), 3,000 into commodity (original), and 2,000 into special/rare. The library has assigned a contributed value factor of 1.0 (a baseline) for commodity (copy) material, a contributed value factor of 3.0 for material processed as commodity (original), and a contributed value factor of 10.0 for the processing of special/rare items 
in the collection to reflect the relative value of these activities (rows 38-40). The authors assume that individual libraries will assign these factors at their own discretion. The basic cost of the four levels of staff who perform this work is recorded in rows 44-47, and the differences among these become the relative cost factors in rows $51-54 .{ }^{17}$ For these values to influence the adjusted acquisitions and cataloging transactions in the right direction (i.e., the lower the cost, the more productive the operation), they are expressed as inverse ratios (rows 51-54, column C) for the purposes of performing the weighting required for the adjusted calculations. Applying all of these inputs formulaically results in 158,592 adjusted acquisitions and cataloging transactions for the target year (row 56, column C).

The values recorded in rows 60-62 reflect the number of FTE allocated to the operations measured for the year in question. The total FTE (row 63) provides one of the key elements in the equation to calculate the efficiency quotient in row 65. In this case, 18 FTE (adjusted to 17.55 for a 40-hour workweek) were required to perform the 158,592 adjusted acquisitions and cataloging transactions, which generates an efficiency quotient of 9.04 (FTE per 1,000 items)..$^{18}$ The model prompts further analysis of these FTE as a percentage of the total number of non-managerial technical services FTE in the operation for the target year (rows 69-71). This percentage of non-managerial FTE (51.4 percent) is compared to the number of adjusted uses of items in physical formats (row 79) as a percentage of the total number of charges, renewals, and downloads of the collection as a whole (18.4 percent) to derive the usage value multiplier in row 83 (3.02). Applying this multiplier to the efficiency quotient yields the alignment indicator (27.33) for the first year measured (row 85, column C).

Considered in isolation, this arbitrarily generated number reveals nothing until the model is applied to a second (or subsequent) set of periodically compiled data. It is necessary to remember that this method of assessment is not intended for the analysis of a single snapshot of data, but as a tool to reflect changes in user behavior, developments (or stagnation) in a library's collecting patterns, and adjustments in technical services staffing alignment in response to these changes and developments. For this reason, it will be useful to examine a second year of sample data, as represented in columns $\mathrm{D}$ and $\mathrm{E}$ of the table.

Keeping in mind the inverted pyramid of the staff alignment assessment methodology in figure 1, the authors note first that the alignment indicator for the second year measured is 29.04 (row 85, column E). This value reflects an increase of 1.71 over the previous year. The allocation of staff in technical services within this fictitious research library now seems better aligned with the library's collection development goals and with its users' interests. The factors influencing this improvement are complex, since

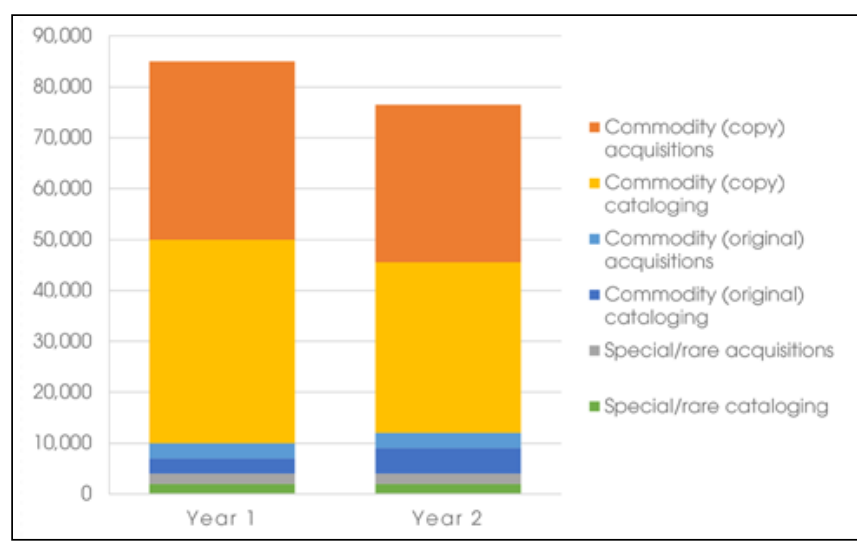

Figure 6. Titles processed by transaction type in Years 1 and 2.

a combination of small and nuanced changes in staff productivity, efficiency, and user behavior contribute to this statistical indicator. The model is designed to help sort this out.

As the notes in column $\mathrm{F}$ of table 1 indicate, the increase in the alignment indicator in Year 2 is the product of a number of noteworthy changes in the variables represented in the table. Among these are a significant decrease in the total number of items in physical formats acquired (row 4), all of which fall into the category of commodity (copy), and a decrease in the total number of items cataloged (row 5). In the latter, the number of commodity (copy) titles processed declined by 16 percent, while the number of commodity (original) titles rose by 67 percent (rows 19 and 24). During this same period, copy catalogers' participation in the acquisitions processing of commodity material and in commodity (original) cataloging ceased (rows 12, 17, and 27 ), and their contributions to commodity (copy) cataloging decreased (row 22). This decrease in overall copy cataloging staff effort is further substantiated by the decrease in cataloging staff allocation during Year 2 (row 62), resulting from the transfer of one FTE of copy cataloger effort to e-resources processing (row 70). The student wage rate also rose in Year 2 (row 44), which negatively affects the relative cost factor for the least expensive labor tier. Finally, collection use statistics for Year 2 reveal a 1 percent decrease in the use of the physical collection and a 10 percent increase in the use of electronic resources (rows 75 and 80). Figures 6,7 , and 8 contain graphic representations of these changes in technical services activity and collection use from Year 1 to Year 2.

The change in the alignment indicator from Year 1 to Year 2 immediately shows an improvement in the alignment of technical services' productivity, efficiency, and staffing allocations with user behavior and the library's strategic aims. However, it is only by (1) digging down into the data that contributes to the derivation of this indicator and (2) 


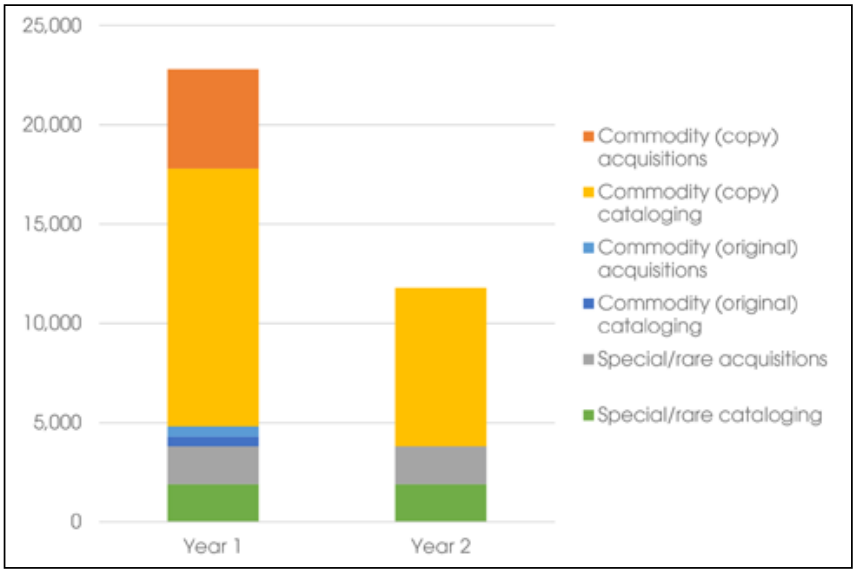

Figure 7. Copy cataloging staff effort by transaction type in Years 1 and 2.

taking into consideration administrative changes that may have effected significant variations in this data from one year to the next, that technical services managers can reach a better understanding of how well their units are performing regarding their libraries' broader goals and why. Such an analysis also reveals how to improve staff alignment further or to correct imbalances in staff alignment when the value of the alignment indicator falls.

\section{Conclusion: Toward Extending the Model}

The authors have sought to introduce a methodology and to demonstrate a prototypical model that technical services managers can use to better assess their unit's productivity and efficiency and the extent to which its activities align with a library's collecting patterns and the behavior of its users using empirical inputs. The model has the potential to reveal both the relative cost-benefit value of the technical services activities measured during the target period and possible misalignments of staff effort or focus outside of technical services. For example, the model might reveal an increase in print acquisitions when the library is purporting to increase its preference for electronic over physical items in its collecting profile and is, consequently, reducing its support for technical staff handling print. In this case, has technical services been able to absorb this unexpected increase by implementing more streamlined or automated workflows? Or is the spike in new items acquired outpacing the rate at which these items are cataloged, leading to cataloging backlogs? Are the latter acceptable? What do the related collection use statistics for the same period reveal?

Readers may have questions about the components of the model. The following questions arose when the authors

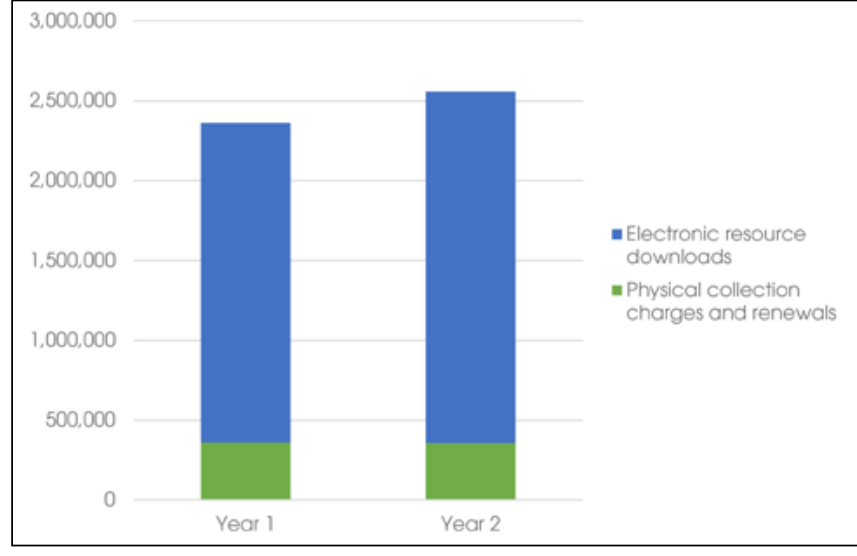

Figure 8. Collection use by resource category in Years 1 and 2.

discussed the model with colleagues: What about physical processing (stamping, spine labeling, security stripping, and barcoding)? Should this effort be included in the model? Is physical processing work a technical services function in the library in question? Or is the activity provided by a preservation unit? What about shelf-ready receipts? Should those titles be counted in the table even if the staff in question do not handle them? The authors believe that they should as long as the shelf-ready arrangement is deemed cost-effective and would improve the assessed alignment of technical services staffing with the library's collection building aims. Should the numbers for collection usage be restricted only to those titles processed during the target period? Are these statistics easy to derive? Is this really the most relevant measurement of collection usage for the purposes of assessing staff alignment? What about the relative difference in collection spending allocated to material in physical versus electronic formats? Should these dollar amounts be included in the model to generate another multiplier (similar to the usage value multiplier) for calculating the alignment indicator?

Such questions clearly point to how the proposed assessment model might be modified or extended into other areas of library operations. Given these and other opportunities for extending the model, the version of the model introduced in this study should not be considered as definitive, but rather as the prototype of a new approach to assessing technical services that invites iteration and adaptation. The authors invite readers to experiment with the model, to adapt it to other functional areas (e.g., electronic resources; non-MARC metadata design and consulting; web archiving; processing of unique, non-rare material stored in institutional repositories), and to deconstruct it in ways that will reveal even more nuanced parameters for measuring the organizational impact of technical services work and the strategic alignment of its staff. 


\section{References and Notes}

1. Roberta Winjum, "Fast and Sleek: Developing Solutions for Technical Services Challenges-A Report of the Technical Services Managers in Academic Libraries Interest Group, American Library Association Annual Conference, Chicago, June 2013," Technical Services Quarterly 31, no. 1 (2014): 67-68.

2. Perhaps the most noteworthy and fruitful of these methodologies were the time and cost studies developed at Iowa State University (ISU) in 1986-87 and 2000-2001, which yielded significant data on departmental productivity and efficiency for later analysis. See, for example, David C. Fowler and Janet Arcand, "Monographs Acquisitions Time and Cost Studies: The Next Generation," Library Resources \& Technical Services 47, no. 3 (2003): 109-24; and David C. Fowler and Janet Arcand, "A Serials Acquisitions Cost Study: Presenting a Case for Standard Serials Acquisitions Data Elements," Library Resources \& Technical Services 49, no. 2 (2005): 107-22. Other institutions, such as Cornell University, adopted the ISU framework for their own cost studies in the 1990s.

3. Cheryl McCain and Jay Shorten, "Cataloging Efficiency and Effectiveness," Library Resources \& Technical Services 46, no. 1 (2002): 23. For an example of one library's workflow/ throughput study, see Patricia Dragon and Lisa Sheets Barricella, "Assessment of Technical Services Workflow in an Academic Library," Technical Services Quarterly 23, no. 4 (2006): 51-72.

4. It has become increasingly common to resort to less empirical methods, such as customer service surveys, focus groups, and other means of gathering input, to evaluate and assess the relationship and impact of technical services activities on user needs and behavior. See, for example, Rebecca L. Mugridge, “Technical Services Assessment: A Survey of Pennsylvania Academic Libraries," Library Resources \& Technical Services 58, no. 2 (2014): 100-110; Rebecca L. Mugridge and Nancy M. Poehlmann, "Internal Customer Service Assessment of Cataloging, Acquisitions, and Library Systems," OCLC Systems \& Services 31, no. 4 (2015): 219-48; and Catherine Sassen, Rebecca Welch, and Kathryn Loafman, "Assessment of Cataloging Services in an Academic Library," Technical Services Quarterly 33, no. 1 (2016): 23-41. The first and third of these articles also contain comprehensive, up-to-date literature reviews related to the assessment of technical services productivity, efficiency, effectiveness, and impact, the repetition of which we will forgo here.

5. Fowler and Arcand, "Monographs Acquisitions Time and Cost Studies," 109.

6. Robert S. Kaplan and Steven R. Anderson, "Time-Driven Activity-Based Costing," Harvard Business Review 82, no. 11 (2004): 131-38.
7. Ibid., 131.

8. Ibid., 133.

9. Although different in methodology from the model introduced here, a group of Belgian librarians have applied Kaplan and Anderson's “TDABC” methodology to an analysis of a library acquisitions operation. See Kristof Stouthuysen et al., "Time-Driven Activity-Based Costing for a Library Acquisition Process: A Case Study in a Belgian University," Library Collections, Acquisitions \& Technical Services 34, no. 2-3 (2010): 83-91. This group emphasizes the importance of assigning tasks to the appropriate level of staff in a unit of this type, an observation that is also germane to the current study.

10. For recent remarks on the importance of this concern, see Mugridge, "Technical Services Assessment," 100; and Sassen, Welch, and Loafman, "Assessment of Cataloging Services in an Academic Library," 23.

11. Matthew Beacom, head of technical services at Yale University's Beinecke Rare Book and Manuscript Library, has characterized this figuration as the "drip coffee assessment pyramid."

12. For instance, see Aiping Chen-Gaffey and Heather Getsay, "More E-books, Less Print?-What Does Usage Data Tell Us?", Library Collections, Acquisitions \& Technical Services 39, no. 3-4 (2015): 59-67. The question of user preference among various formats within different disciplines and in different circumstances remains open-see Lee A. Cummings, Anne Larrivee, and Leslie Vega, "Comparing Electronic vs. Print Book Preferences between Students in the Social Sciences, the Arts, and STEM," Library Hi-Tech News 32, no. 4 (2015): 1-4.

13. Lorcan Dempsey, Constance Malpas, and Brian Lavoie, "Collection Directions: The Evolution of Library Collections and Collecting," portal: Libraries and the Academy 14, no. 3 (2014): 402. In addition to rare material per se (i.e., items held in special collections), Dempsey, Malpas, and Lavoie include another type of unique library holding in their taxonomy of collections: research and learning materials and institutional records created locally (394). This portion of the collection falls outside the scope of the current study, but would be relevant to an institution that processes such material as part of its monograph cataloging workflow or to a model for assessing other technical services functions, such as non-MARC metadata processing. This category of material would likely warrant its own contributed value factor.

14. For a related work that seeks to align library collection building with user behavior, see Julie Linden, Sarah Tudesco, and Daniel Dollar, "Collections as a Service: A Research Library’s Perspective," College \& Research Libraries 79, no. 1 (2018): 86-99, https://doi.org/10.5860/crl.79.1.86. The juxtaposition of physical format and electronic format usage 
data referenced in that study informed our development of the usage value multiplier as applied here (93).

15. The usage value multiplier is expressed as an inverse ratio because as the difference between the staff allocation percentage and the use of the physical collection declines, the alignment indicator must increase inversely to reflect the positive effect of this decrease.

16. In the example, we have not included the acquisition of new serial titles in the count, given the significantly different nature of serials management work from that of monographs. This is an arbitrary choice, as is the inclusion and precise definition of all the variables in columns $\mathrm{B}$ and $\mathrm{D}$ of the table, which are at the discretion of the individual institution using the instrument.
17. There are obviously multiple ways to establish the basic costs for each level of staff represented in the model, including the inventory of how much the institution pays each staff member, plus benefits, then converting these numbers to cost per FTE and deriving an average. However, to permit easy implementation and reapplication of the instrument, we recommend a simpler method, such as the use of hiring minimums or maximums, including benefits, for each level of staff.

18. For some institutions, the conversion of staff hours to a standard work week may not be necessary. However, in situations where the FTE definitions for staff levels vary (e.g., when the length of the standard workweek for hourly staff is different from that of salaried staff), this conversion increases the accuracy of the measurements. 